\title{
Acceptance and market for ADAS and automated driving systems
}

Dr. Wolfgang Bernhart, M. Yoon, Roland Berger GmbH

This manuscript is not available according to publishing restriction.

Thank you for your understanding. 